\title{
Circulating blood levels of IL-6, IFN- $\gamma$, and IL-10 as potential diagnostic biomarkers in gastric cancer: a controlled study
}

\author{
Norma Sánchez-Zauco 1,2,3, Javier Torres ${ }^{4}$, Alejandro Gómez ${ }^{4}$, Margarita Camorlinga-Ponce ${ }^{4}$, \\ Leopoldo Muñoz-Pérez ${ }^{4}$, Roberto Herrera-Goepfert ${ }^{5}$, Rafael Medrano-Guzmán ${ }^{6}$, Silvia Giono-Cerezo ${ }^{3}$ \\ and Carmen Maldonado-Bernal ${ }^{1 *}$ (D)
}

\begin{abstract}
Background: Gastric adenocarcinoma is the third most common cause of cancer-associated death worldwide. Helicobacter pylori infection activates a signaling cascade that induces production of cytokines and chemokines involved in the chronic inflammatory response that drives carcinogenesis. We evaluated circulating cytokines and chemokines as potential diagnostic biomarkers for gastric cancer.

Methods: We included 201 healthy controls and 162 patients with distal gastric cancer who underwent primary surgical resection between 2009 and 2012 in Mexico City. The clinical and pathological data of patients were recorded by questionnaire, and the cancer subtype was classified as intestinal or diffuse. Pathological staging of cancer was based on the tumor-node-metastasis staging system of the International Union Against Cancer. Concentrations of IL-1 $\beta, I L-6$, TNF- $\alpha, I L-10$, and MCP-1 in serum were measured using multiplex analyte profiling technology and concentrations of IL-8, IFN- $\gamma$, and TGF- $\beta$ in plasma were measured using enzyme-linked immunosorbent assay.

Results: Levels of IL-1 $\beta, I L-6$, IFN- $\gamma$, and IL-10 were significantly higher and that of MCP-1 was lower in gastric cancer patients compared with controls. No differences in IL-8 or TNF-a levels were observed between gastric cancer and controls. IFN- $\gamma$ and IL-10 were significantly higher in both intestinal and diffuse gastric cancer, whereas IL-1 $\beta$ and IL- 6 were higher and TGF- $\beta$ lower only in intestinal gastric cancer; MCP- 1 was lower only in diffuse gastric cancer. IFN- $\gamma$ and IL-10 levels were significantly higher in early (I/II) and late stage (III/IV) gastric cancer; IL-1 $\beta$ and IL8 were higher and MCP-1 was lower only in late stage (IV) patients. Receiver-operating characteristic analysis showed that for diagnosis of GC, IL-6 had high specificity (0.97) and low sensitivity (0.39), IL-10 had moderate specificity (0.82) and low sensitivity (0.48), and IL-1 $\beta$ and IFN- $\gamma$ showed low specificity (0.43 and 0.53 , respectively) and moderate sensitivity ( 0.76 and 0.71 , respectively).
\end{abstract}

Conclusions: Increased levels of IL-6, IFN- $\gamma$, and IL-10 might be useful as diagnostic biomarkers for GC; however, this needs to be confirmed with larger number of patients and with control groups other than blood donors, properly age paired. IL-1 $\beta$, IL-6, MCP-1, and TGF- $\beta$ differentiate intestinal from diffuse GC. IFN- $\gamma$ and IL-10 might be useful for diagnosis of early stage GC, and IL-1 $\beta, I L-8$, and MCP-1 for late stages of the disease.

Keywords: Gastric cancer, Biomarker, Inflammatory cytokines, Immunosuppressive mediators, Helicobacter pylori

\footnotetext{
* Correspondence: cmaldobe@yahoo.com

'Laboratorio de Investigación en Inmunología y Proteómica, Hospital Infantil

de México Federico Gómez, Dr. Márquez 162, Col. Doctores, 06720 Mexico

City, Mexico

Full list of author information is available at the end of the article
} 


\section{Background}

Gastric cancer (GC) remains a major health problem worldwide, representing the third most common cause of death from all cancers [1]. Mortality rates are higher in Asian and Latin American countries, where cases are usually diagnosed at later stages, leading to very low survival rates. The infection of the gastric mucosa by Helicobacter pylori represents the major risk factor in over $65 \%$ of all distal GC, and recent evidence suggests that it might also play a role in proximal GC $[2,3]$. H. pylori has the ability to colonize the human stomach and persist for decades, eliciting a chronic long-lasting inflammatory response that varies in magnitude depending on the genetic background of the host, the virulence of the $H$. pylori strain, and environmental factors [4]. The differences in the gastric inflammatory response between hosts may partially explain the different outcomes observed in $H$. pylori-infected patients [5]. Understanding the natural history of the infection may help to identify potential biomarkers for the early diagnosis of GC to allow timely treatment to reduce mortality.

The interaction of $H$. pylori with the gastric epithelium induces the production of interleukin (IL)-8, IL-6, and IL$1 \beta$. These cytokines are chemotactic for neutrophils and mononuclear cells, and their production leads to a proliferative response with a dense infiltrate of neutrophils and macrophages in the gastric mucosa, resulting in a chronic active gastritis [6]. H. pylori also induces gastric mucosal infiltration by dendritic cells and T and B cells, and stimulates secretion of macrophage chemotactic protein (MCP)-1, tumor necrosis factor (TNF)- $\alpha$, IL-12, IL-10, transforming growth factor (TGF)- $\beta$, and interferon (IFN)- $\gamma$ [5]. Among other deleterious effects that predispose cells to oncogenic transformation, the inflammatory mediators produced by this decades-long gastritis may cause DNA damage, induce proliferation, and inhibit apoptosis [7]. In addition, inflammatory cytokines and chemokines increase the expression of molecular factors such as hypoxia-inducible factor-1, vascular endothelial growth factor, L-selectin, cyclooxygenase-2, and matrix metalloproteinase together with other molecules that contribute to carcinogenesis [7]. Progress of GC can be evaluated using the tumor-nodemetastasis (TNM) staging classification of malignant tumors, which is an important prognostic factor for GC [8]. Ideally, biomarkers should detect the early stages (I/II) of disease when opportunities for cure are highest.

The inflammatory mediators produced locally in the gastric mucosa may reach the blood circulation and be detected in plasma samples. In this study, we tested the hypothesis that circulating levels of inflammatory cytokines and chemokines could function as indirect indicators of tissue damage, and that their measurement might be a useful biomarker for the early detection of $\mathrm{GC}$, resulting in a better long-term prognosis.

\section{Methods}

\section{Study population}

The study included 166 patients with GC who attended the Oncology Hospital, Centro Médico Nacional Siglo XXI, Instituto Mexicano del Seguro Social (IMSS) and the Instituto Nacional de Cancerología, Secretaría de Salud in Mexico City, and who underwent primary surgical resection between 2009 and 2012. Patients $\leq 18$ years old, with any autoimmune disease, diabetes, cancer other than GC, or having received previous treatment for GC were excluded from the study. Informed written consent was obtained from all patients prior to enrollment in the study. The Ethics Committees of the two participating institutions, IMSS and Secretaría de Salud, approved the study. The clinical and pathological data of patients were recorded using a questionnaire, and the cancer subtype was classified as intestinal GC (IGC) or diffuse GC (DGC) based on the Lauren classification [9]. Pathological staging of cancer was according to the TNM staging system of the International Union Against Cancer American Joint Committee on Cancer 2010. The study included as control group 201 healthy adults recruited in 2011-2012 (within the same period of time as for GC cases), and who were blood donors at the Central Blood Bank, Centro Médico Nacional Siglo XXI, IMSS. GC cases and healthy controls were of the same community, with similar socioeconomic and demographic conditions.

\section{Blood samples}

Patients were asked to provide a blood sample before surgery and prior to any cancer treatment. Blood was drawn after overnight fasting into either ethylenediaminetetraacetic acid or plain tubes. The plasma or serum samples were stored at $-20{ }^{\circ} \mathrm{C}$ until tested.

\section{Status of $H$. pylori infection}

Sera of patients were tested for antibodies against $H$. pylori whole-cell antigens and against CagA protein, using a previously validated enzyme-linked immunosorbent assay (ELISA). H. pylori infection was defined as positive when antibodies to whole-cell antigens and/or to CagA protein were present.

\section{Cytokine assays}

In the first part of the study, we tested plasma samples for IFN- $\gamma$, IL-8, and TGF- $\beta$; in the second part, we tested in addition for IL-10, IL-1 $\beta$, TNF- $\alpha$, IL-6, and MCP-1 in serum (see Additional file 1: Table S1).

The concentration of IL- 8 , IFN- $\gamma$, and TGF- $\beta$ in plasma samples was measured by ELISA using commercially available kits (BD ${ }^{\text {ts }}$ OptEIA; BD Biosciences, Rockville, $\mathrm{MD})$ according to the manufacturer's instructions. The concentration of cytokine or chemokine was calculated 
based on standard curves provided with the kits, and results were expressed in $\mathrm{pg} / \mathrm{ml}$.

Concentrations of IL-1 $\beta$, IL-6, TNF- $\alpha$, IL-10, and MCP-1 were measured with multiplex analyte profiling technology (xMAP) using the Merck-Millipore, USA HSCYTOMAG-60 K Kit (Luminex ${ }^{\circ}$, magnetic beads Millipore, Billerica, MA) read on MAGPIX equipment (MILLIPLEX ${ }^{\circ}$, Millipore). The assay kits included a standard curve and two controls (high and lower concentration) for each cytokine. For both ELISA and xMAP platforms, all samples were tested in duplicate and the average values were used in the analysis.

\section{Statistical analysis}

Values were expressed as median with interquartile ranges, and the comparison between groups was performed using Kruskal-Wallis and Mann-Whitney $U$ tests, $p<0.05$ was considered to indicated a statistically significant result. The association of each cytokine with GC was assessed using odds ratio (OR) and 95\% confidence interval (CI). The results for cytokines were adjusted for patient age and sex by multivariate logistic regression analyses. A cutoff value was determined for each cytokine using a receiver-operating characteristic (ROC) curve, (Additional file 1: Table S2) and each value was used to estimate the sensitivity, specificity, positive predictive value (PPV), and negative predictive value (NPV) with 95\% CI. All statistical analyses were performed using IBM SPSS Statistics software (v. 20, IBM Corp., Armonk, NY).

\section{Results}

The characteristics of the patients and healthy donors included in the study are described in Table 1 . The study included 363 individuals, of whom 162 (44.6\%) were patients with GC. The group with cancer had an average age almost twice that of the healthy donor (control) group $(p<0.05)$; the ratio of females and males was similar. The frequency of seropositivity for $H$. pylori was significantly higher in the GC group than in the control group $(p<0.05)$; H. pylori infection showed an OR of 2.29 (1.473.58) for risk of developing GC. DGC was more prevalent than IGC $(p<0.05)$, and tended to occur at a younger age than IGC. Other histological types of GC represented only $22.8 \%$ of the studied sample. Only 29\% of CG cases were classified as stage I or II by TNM; the majority of cases (63\%) were stage III or IV. The observed female:male ratio and frequencies of GC subtypes were consistent with the recently described epidemiology of GC in Mexico [10-12].

\section{Circulating levels of IL-1 $\beta$, IL- 6 , IFN- $\gamma$, IL-10, and MCP-1 but not IL-8, TNF- $\alpha$, or TGF- $\beta$ differentiate GC patients from healthy controls}

Circulating levels of IL-1 $\beta$, IL-6, IFN- $\gamma$, and IL-10 were significantly higher and levels of MCP-1 were significantly lower in GC patients than in the healthy control group (Fig. 1). In contrast, levels of IL- 8 and TNF- $\alpha$ did not differ significantly between GC patients and healthy controls (Additional file 2: Fig. S1). However, we noted that 22 patients in the GC group (of 143, 15.4\%) had IL-8 concentrations $\geq 90 \mathrm{pg} / \mathrm{ml}$, whereas in the healthy group only four individuals (of 121 , $3.3 \%)$ had concentrations this high; this difference was significant $(p=0.0154)$ (Additional file 2: Fig. S1B).

The ORs for all cytokines and chemokines confirmed that elevated IL-1 $\beta$, IL- 6 , IFN- $\gamma$, and IL-10 were risk factors for GC (Table 2).

We subsequently performed a multivariate logistic regression analysis adjusting for age and sex, and found that after adjustment IL-6, IFN- $\gamma$, IL-10, and MCP-1 had significant associations with GC (Table 3). Thus, after correcting for age, three (IL-6, IFN- $\gamma$, and IL-10) of the four cytokines associated with GC in the univariate analyses still maintained a significant association with the disease.

\section{Analyses of circulating cytokines by type of GC reveal differences between IGC and DGC}

We next analyzed cytokine levels in the DGC and in IGC subgroups compared with the control group, and found important differences. IFN $-\gamma$ and IL-10 were significantly higher in both DGC and IGC patients (Fig. 1) than in controls. However, in contrast to the results obtained for the whole group of GC patients, we found that IL- $1 \beta$ and IL- 6 levels were significantly higher in the IGC group but not in the DGC group, and that levels of MCP-1 were significantly lower in the DGC group but not in the IGC group. In

Table 1 Characteristics of the gastric cancer patients and healthy blood donors included in the study

\begin{tabular}{lllll}
\hline Group & No. tested (\%) & Age Average \pm SD & Ratio male: female & $\%$, H. pylori + \\
\hline Healthy donors & 201 & $35.2 \pm 11.1$ & 1.01 & 53.0 \\
Gastric Cancer & 162 & $62.1 \pm 13.2$ & 0.93 & 72.3 \\
Subtype & & & 78.4 \\
$\quad$ Diffuse & $76(46.9)$ & $57.8 \pm 12.6$ & 0.68 & 59.2 \\
Intestinal & $49(30.2)$ & $60.2 \pm 11.8$ & 0.92 & 76.7 \\
Mixed & $30(18.5)$ & $63.6 \pm 13.7$ & 1.21 & 83.3 \\
$\quad$ Other & $7(4.3)$ & $59.6 \pm 11.5$ & 7.0 & \\
\hline
\end{tabular}



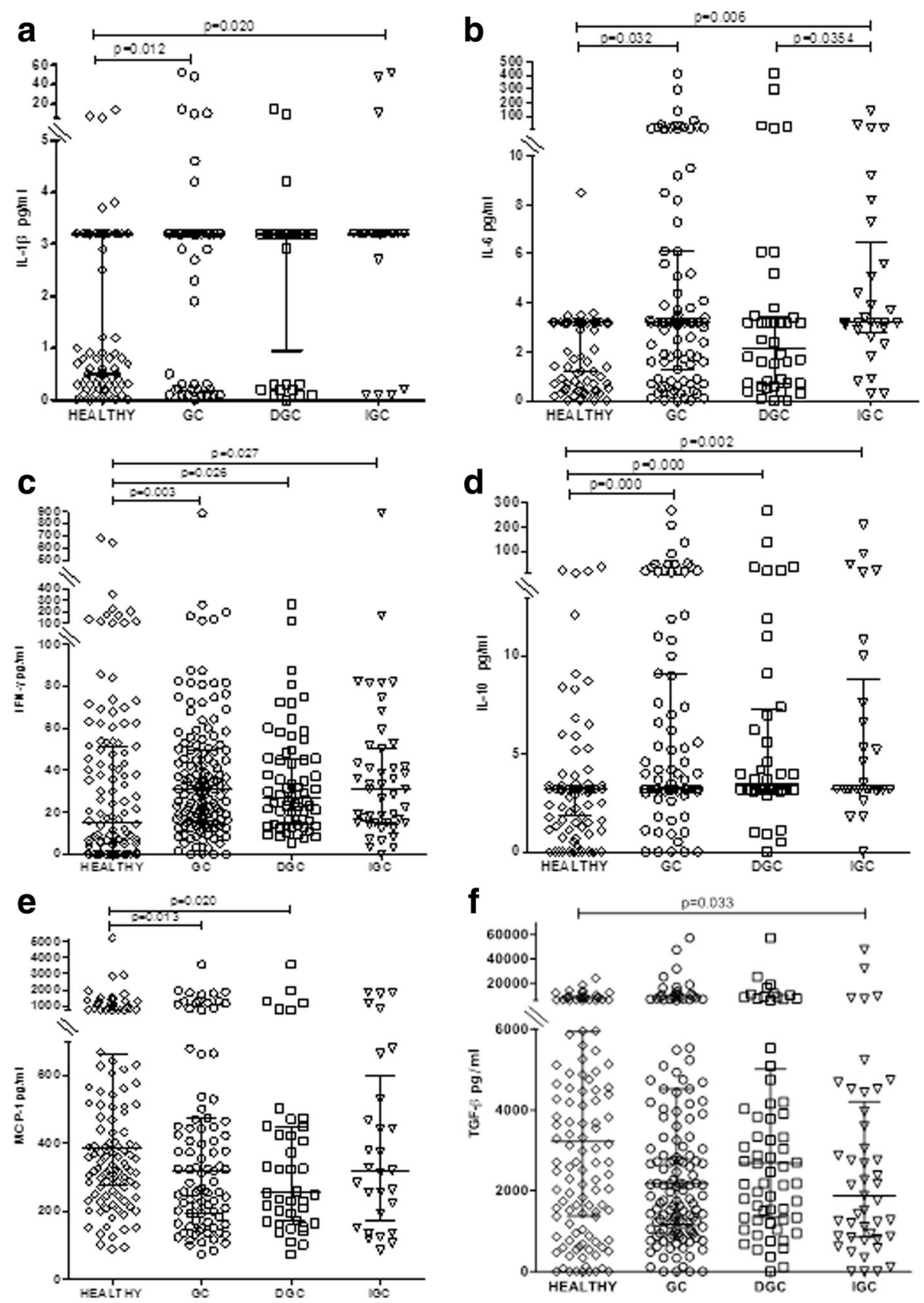

Fig. 1 The circulating concentrations of IL-1 $\beta, I L-6, I F N-\gamma, I L-10, M C P-1$, and TGF- $\beta$ differ between healthy controls and gastric cancer patients. Circulating concentrations of IL-1 $\beta$ (a), IL-6 (b), IFN- $\gamma(\mathbf{c})$, IL-10 (d), MCP-1 (e), and TGF- $\beta$ (f) in healthy controls and patients with gastric cancer were measured by ELISA or XMAP. The gastric cancer group was divided into diffuse (DGC) and intestinal (IGC) types. All measurements were made in duplicate. The statistical analysis was performed by Mann-Whitney $U$ test and the results for each group are presented as median with interquartile range

addition, levels of TGF- $\beta$ were significantly lower than controls in the IGC group but not in the DGC group (Fig. 1). These results were confirmed in a multivariate logistic regression analyses after adjusting for age and sex.
Analyses of circulating cytokines show important differences between TNM stages of GC

We analyzed cytokine levels in patients with different stages of GC compared with healthy controls and found that concentrations of IFN- $\gamma$ and IL-10 were significantly higher in 
Table 2 Odds ratio and $p$-values for the cytokines and chemokines evaluated as risk factor for gastric cancer

\begin{tabular}{|c|c|c|c|c|c|}
\hline Cytokine & $\begin{array}{l}\text { Positive } \\
(\%)\end{array}$ & $\begin{array}{l}\text { Negative } \\
(\%)\end{array}$ & OR & IC 95\% & $p$-value \\
\hline \multicolumn{6}{|l|}{$\mathrm{IL}-1 \beta$} \\
\hline Healthy & $65(49)$ & $50(70)$ & & & \\
\hline Gastric cancer & $68(51)$ & $21(30)$ & 2.49 & $1.35-4.59$ & 0.003 \\
\hline \multicolumn{6}{|l|}{ IL-6 } \\
\hline Healthy & $4(10)$ & $111(68)$ & & & \\
\hline Gastric cáncer & $35(90)$ & $54(32)$ & 17.9 & $6.08-53.19$ & 0.00 \\
\hline \multicolumn{6}{|l|}{ IFN $-\gamma$} \\
\hline Healthy & $56(35)$ & $64(61)$ & & & \\
\hline Gastric cancer & $101(65)$ & $42(39)$ & 2.75 & $1.65-4.57$ & 0.00 \\
\hline \multicolumn{6}{|l|}{ IL-10 } \\
\hline Healthy & $21(36)$ & $94(67)$ & & & \\
\hline Gastric cancer & $43(64)$ & $46(33)$ & 4.18 & $2.23-7.85$ & 0.00 \\
\hline \multicolumn{6}{|l|}{ MCP-1 } \\
\hline Healthy & $59(60)$ & $56(50)$ & & & \\
\hline Gastric cancer & $38(40)$ & $53(50)$ & 0.68 & $0.39-1.19$ & 0.21 \\
\hline \multicolumn{6}{|l|}{ IL-8 } \\
\hline Healthy & $49(40)$ & $71(50)$ & & & \\
\hline Gastric cancer & $71(60)$ & $71(50)$ & 1.45 & $0.89-2.37$ & 0.17 \\
\hline \multicolumn{6}{|l|}{ TNF-a } \\
\hline Healthy & $82(57)$ & $33(58)$ & & & \\
\hline Gastric cancer & $63(43)$ & $28(42)$ & 0.91 & $0.49-1.65$ & 0.76 \\
\hline \multicolumn{6}{|l|}{ TGF- $\beta$} \\
\hline Healthy & $64(52)$ & $56(40)$ & & & \\
\hline Gastric cancer & $58(48)$ & $83(60)$ & 0.61 & $0.37-0.99$ & 0.06 \\
\hline
\end{tabular}

the early stages (I/II) of GC and remained higher in late stage (IV) GC (Fig. 2c and d). However, concentrations of IL-1 $\beta$ and IL- 8 were significantly higher and that of MCP-1 significantly lower in patients with stage IV GC (Fig. 2a, e, and f) compared with controls, but not in patients with early stage GC. Levels of TNF- $\alpha$ were not significantly different to controls at any stage of GC (Fig. 2g). Concentrations of IL- 6 were significantly higher and those of TGF- $\beta$

Table 3 Multivariate Logistic regression analysis for cytokines associated with risk for gastric cancer, adjusted by age and gender

\begin{tabular}{lllll}
\hline Cytokines & OR & \multicolumn{2}{l}{$95 \% \mathrm{Cl}$} & $p$-value \\
\cline { 3 - 4 } & & Lower & Upper & \\
\hline IL-1 $\beta$ & 1.89 & 0.75 & 4.77 & 0.178 \\
IL-6 & 8.46 & 2.04 & 35.04 & 0.003 \\
IFN- $\gamma$ & 3.02 & 1.35 & 6.78 & 0.007 \\
IL-10 & 5.24 & 1.95 & 14.09 & 0.001 \\
MCP-1 & 0.33 & 0.13 & 0.81 & 0.016 \\
\hline
\end{tabular}

$p<0.05$ statistical significance were lower than controls in patients with late stage III GC (Fig. 2b and h). Multivariate logistic regression analyses confirmed these results after adjusting for age and sex.

Sensitivity and specificity suggest that IL-6, IFN- - , and IL10 might be useful for differentiating patients with GC

Because association analyses are not sufficient to identify possible diagnostic markers, we next determined cutoff values for each cytokine using ROC analysis, which showed moderate values for the areas under the curve (described in Additional file 1: Table S2). Using these cutoff values, we explored the possible utility of these cytokines as a diagnostic test (Table 4). IL-6 showed good specificity (0.97) and low sensitivity (0.39), IFN- $\gamma$ showed low specificity (0.53) and moderate sensitivity (0.71), and IL-10 showed moderate specificity (0.82) and low sensitivity (0.48), suggesting that these cytokines might be of value for identifying patients with GC. In contrast, IL-1 $\beta$ and MCP- 1 showed poor specificity $(0.43$ and 0.49 , respectively), eliminating them as potentially useful diagnostic biomarkers.

\section{Discussion}

We analyzed the systemic levels of six cytokines and two chemokines associated with chronic gastric inflammation during $H$. pylori infection for potential biomarkers to identify patients with GC. We found that increased levels of IL-1 $\beta$, IL-6, IFN- $\gamma$, and IL-10 and lower levels of MCP-1 significantly differentiated patients with GC from healthy controls. We also classified the GC cases according to their TNM stage and found that higher levels of IL-1 $\beta$, IFN- $\gamma$, IL-10, and IL-8, and lower levels of MCP-1 differentiated late stage IV GC from healthy controls, and importantly, that IFN- $\gamma$ and IL-10 differentiated patients with early stage (I/II) GC from healthy controls. These results suggest that circulating levels of cytokines may help to identify patients in the early stages of GC, offering the possibility of early detection for timely treatment.

The observed differences of these cytokines between GC patients and healthy controls are consistent with reports in both animal models and human infection. For example, IL-1 $\beta$ is a proinflammatory cytokine required for the efficient control of $H$. pylori infection [13]. IL-1R $(-/-)$ mice failed to develop protective immunity but were protected against Helicobacter-associated gastritis and gastric preneoplasia because of their inability to generate Helicobacter-specific Th1 and Th17 responses [14]. In addition, the overexpression of IL-1 $\beta$ in the stomach of mice led to spontaneous gastric inflammation and cancer, even in the absence of $H$. pylori infection [15], and IL-1 $\beta$ is a potent inhibitor of gastric acid secretion [16], which may also favor the appearance of preneoplastic lesions because of hypochloridia. 
a

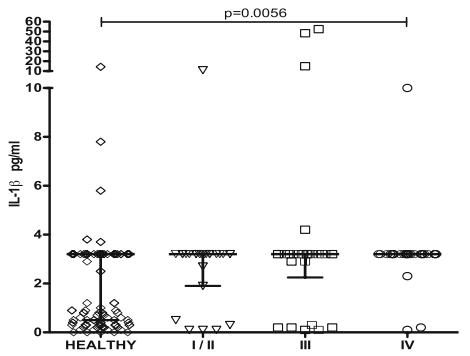

C

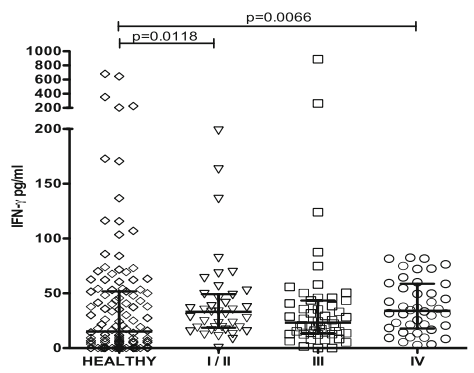

e

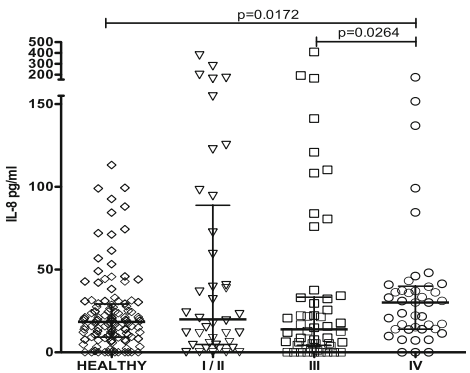

g

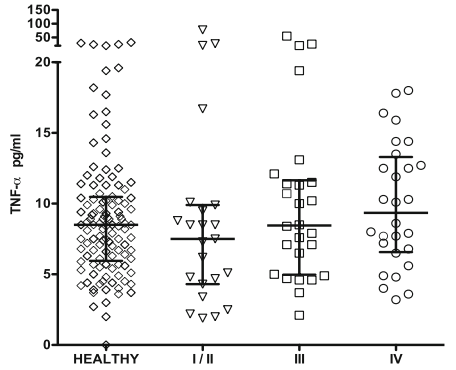

b
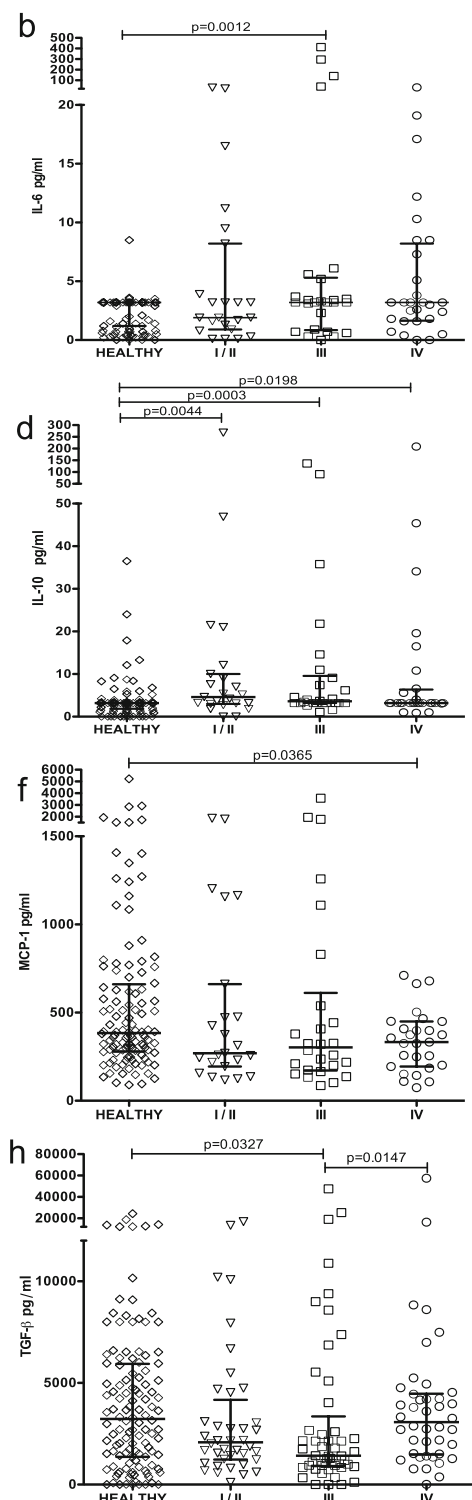

Fig. 2 Analyses of circulating cytokines by TNM stage of GC show important differences between different stages of GC and healthy controls.

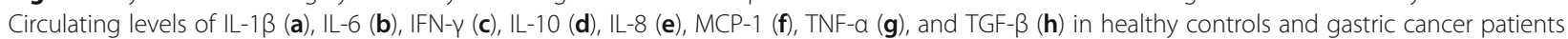
classified according to the TNM staging as stage I/II, III, or IV were measured by ELISA or XMAP. All measurements were made in duplicate. The statistical analysis was performed by Mann-Whitney $U$ test and results for each group are presented as median with interquartile range

The increased levels of IL-6 in GC patients and their strong association with the risk of GC that was identified in this study indicate that IL-6 plays an important role in $\mathrm{GC}$, which is consistent with previous reports in animal models and in patients [16, 17]. IL-6 can upregulate DNA methyltransferases, resulting in modification of the methylation status of genes associated with tumor suppression [18]. In a mouse model of colitis-associated cancer, IL-6 stimulated the survival and proliferation of premalignant intestinal epithelial cells, mainly because of increased signal transducer and activator of transcription (STAT) 3 signaling [19]. It has also been observed that IL-6 negatively affects the expression of genes associated with tumor suppression and anchorage-dependence growth in colonic epithelial cells in vitro [19]. Furthermore, it was reported that in patients with GC, expression of IL-6 and STAT3 was increased in GC tumor tissue and that the level was associated with the TNM stage of GC [20].

The higher level of IFN- $\gamma$ in sera of GC patients compared with controls in this study could be indicative of its role in establishing a proinflammatory microenvironment in the gastric tissue, although its role in the promotion of GC is still controversial. IFN- $\gamma$ is upregulated in the gastric mucosa after chronic $H$. pylori infection 
Table 4 Sensitivity and specificity estimates for each cytokine as a diagnostic test for gastric cancer

\begin{tabular}{|c|c|c|}
\hline \multicolumn{3}{|l|}{$\mathrm{IL}-1 \beta$} \\
\hline Sensitivity & 0.76 & $0.68-0.85$ \\
\hline Specificity & 0.43 & $0.34-0.53$ \\
\hline PPV & 0.51 & $0.42-0.59$ \\
\hline NPV & 0.70 & $0.61-0.82$ \\
\hline \multicolumn{3}{|l|}{ IL-6 } \\
\hline Sensitivity & 0.39 & $0.29-0.49$ \\
\hline Specificity & 0.97 & $0.93-1.00$ \\
\hline PPV & 0.90 & $0.80-0.99$ \\
\hline NPV & 0.67 & $0.60-0.74$ \\
\hline \multicolumn{3}{|l|}{ IFN-Y } \\
\hline Sensitivity & 0.71 & $0.63-0.78$ \\
\hline Specificity & 0.53 & $0.44-0.62$ \\
\hline PPV & 0.64 & $0.57-0.72$ \\
\hline NPV & 0.60 & $0.51-0.70$ \\
\hline \multicolumn{3}{|l|}{ IL-10 } \\
\hline Sensitivity & 0.48 & $0.38-0.59$ \\
\hline Specificity & 0.82 & $0.75-0.89$ \\
\hline PPV & 0.67 & $0.56-0.79$ \\
\hline NPV & 0.67 & $0.59-0.75$ \\
\hline \multicolumn{3}{|l|}{ MCP-1 } \\
\hline Sensitivity & 0.42 & $0.32-0.52$ \\
\hline Specificity & 0.49 & $0.40-0.58$ \\
\hline PPV & 0.39 & $0.29-0.49$ \\
\hline NPV & 0.51 & $0.42-0.61$ \\
\hline
\end{tabular}

PPV positive predictive value

$N P V$ negative predictive value

[21-24], and besides its role in the responses to bacterial infection, it has also an important tumor suppressor activity [22]. Previous studies have shown that specific T cell responses play a critical role in inducing gastric mucosal inflammation $[23,24]$, and suggested that IFN- $\gamma$ may exacerbate gastric inflammation and favor progression to GC. However, more recent studies in a mouse model found that overexpression of IFN- $\gamma$ inhibited gastric carcinogenesis induced by IL-1 $\beta$ and/or Helicobacter infection by suppressing putative gastric progenitor cell expansion and by reducing epithelial cell apoptosis via induction of an autophagy program [25]. Thus, IFN- $\gamma$ is a pleiotropic mediator with both pro- and antitumorigenic activities. IL-10 is also a potent pleiotropic cytokine that has the dual ability to suppress or stimulate anticancer activity [26]. The high amount of IL-10 found in GC patients in this study would suggest that it is acting by suppressing anticancer responses. In fact, when IL-10 is elevated in blood during advanced GC, it leads to the inability to eliminate tumor cells $[27,28]$. GC cells themselves can also secrete IL-10, which may explain its reduction in GC patients after surgical removal of their tumor $[27,28]$.

In contrast to the above cytokines, we found significantly decreased levels of MCP- 1 in the sera of patients with GC. This chemokine plays an important role in the progress of $H$. pylori-related gastric diseases [29], and patients infected with $H$. pylori have significantly higher expression of $m c p-1$ mRNA in the gastric mucosa than patients without $H$. pylori infection [30]. MCP-1 is a CC chemokine that is produced by gastric epithelial cells; it plays a major role in regulating migration of monocytes and lymphocytes into tissues [30] and may also promote angiogenesis and recruitment of tumor-associated macrophages [31,32]. There are few studies reporting the circulating levels of MCP-1 in patients with GC, although one study reported significantly lower levels in cancer patients than in controls [29], which is consistent with our findings. Furthermore, the authors of that study reported that the concentration of MCP-1 in the serum of GC patients decreased in conjunction with disease progression and suggested that reduced plasma levels reflect the local consumption of MCP-1 by GC tissue [29].

We found no significant differences in the levels of circulating IL- 8 , TNF- $\alpha$, and TGF- $\beta$ between GC patients and healthy controls. The lack of significant differences in IL-8 contrasts with previous studies where circulating IL-8 levels were found to be increased significantly in patients who developed GC [33, 34]. Although we found no overall difference in IL-8 levels between the GC and healthy control groups, in GC patients there was a significantly higher frequency of IL-8 values in the upper quartile compared with controls. In addition, patients with late stage (IV) GC had significantly higher levels of circulating IL-8. These results are consistent with studies showing that IL-8 is also produced by cancer cells and may promote angiogenesis, tumor growth, tissue invasion, and metastatic spread [34, 35 , and that high IL-8 expression directly correlates with a poor prognosis in GC [36]. TNF- $\alpha$ is one of the main mediators of inflammation and has been linked to several steps involved in carcinogenesis, including cellular transformation, survival, proliferation, invasion, angiogenesis, and metastasis [37]. However, similar to previous reports [33], we found no differences in TNF- $\alpha$ levels between GC patients and healthy controls. Although TGF- $\beta$ can also promote tumor growth, invasion, and metastasis, it has pleiotropic activity and functions as a tumor suppressor during the early stages of $\mathrm{GC}$, although it may promote tumor growth and metastasis during late stage GC [38].

We then analyzed the differences in cytokines and chemokines between the two types of GC (DGC and IGC) and found that IFN- $\gamma$ and IL-10 were increased significantly in both types relative to controls. In contrast, IL-1 $\beta$, IL-6, and TGF- $\beta$ significantly differentiate IGC but not DGC, whereas MCP-1 was significantly 
lower in DGC but not in IGC. These results suggest that the pattern of cytokine production associated with GC may differentiate DGC from IGC. The lack of studies analyzing circulating cytokines in the different GC types precludes any comparison, and identifies a need for additional studies to validate our results in larger groups of patients and in different populations.

To evaluate further the utility of these markers for differentiating patients with GC from controls, we next determined the ORs and confirmed that increased levels of IL$1 \beta$, IL-6, IFN- $\gamma$, and IL-10 were all associated with increased risk of GC, noting that high levels of IL-6 increased the risk of current GC 17.9 times. The analyses also showed no increase in risk associated with MCP-1, IL-8, and TNF$\alpha$, whereas there was a trend to an association of diminished TGF- $\beta$ with a decreased risk of GC. This analysis confirmed the possible value of IL- $1 \beta$, IL-6, IFN- $\gamma$, and IL10 for differentiating patients with GC in the population studied and suggested that TGF- $\beta$ may also help in the identification of GC risk. We then tested the utility of these markers as a diagnostic test and found that only IL-6, IFN$\gamma$, and IL-10 had a useful specificity $(0.97,0.53$, and 0.82 respectively) and PPV (0.90, 0.64, and 0.67, respectively), but with a low sensitivity $(0.39,0.71$, and 0.48 , respectively). The other cytokines evaluated had poor value as a diagnostic test. Although a number of studies report the association of inflammation markers with GC, none reports the value of circulating levels of markers as a diagnostic test for GC. Such an analysis is needed for better evaluation of the utility of candidate biomarkers in GC.

In the evaluation of possible biomarkers, there is a need for consensus about the type of analyses that are required to define their utility for the timely diagnosis of patients with GC. Some authors limit their analysis to a description of the differences in candidate markers between GC and controls and report p-values, whereas others go further and report ORs; however, few studies test the utility of markers as a diagnostic test and report specificity, sensitivity, and predictive values. In this work, we present sequential analyses of the differences, the ORs, and diagnostic utility of circulating levels of cytokines and chemokines in patients with GC to compare the relevance of the different analytical approaches.

A consistent finding for all cytokines and chemokines tested in this study was the high level of variation between individuals in circulating cytokine levels, which, importantly, limits their possible value as candidate GC biomarkers. Another limitation of our study was that the group with GC had an average age almost twice as high as that of the healthy blood-donor control group; and although the multivariate logistic regression analyses adjusted for age confirmed association with GC, results need to be confirmed with control groups adjusted by age and sex. It is also true that despite the investment of hundreds of millions of dollars over the last 10 years in studies of cancer, none of the analyzed biomarkers has yet been approved for clinical use [39]. However, in view of the urgent need for biomarkers in GC and the lack of validated candidates, efforts to identify possible markers are badly needed, particularly in regions like Latin America that have the highest GC mortality rates. Thus, despite their poor sensitivity, our results for IL-6, IFN- $\gamma$, and IL-10 might offer a limited but still valuable test with reasonable specificity and PPV to identify patients with GC in high-risk regions.

\section{Conclusions}

Our results suggest that high circulating levels of IL-6, IFN- $\gamma$, and IL-10 might be associated with GC, and may be potentially useful as biomarkers to identify patients at risk for GC. We also found that levels of IL-1 $1 \beta$, IL-6, MCP- 1 , and TGF- $\beta$ might be useful to differentiate between IGC and DGC, and that high levels of IFN- $\gamma$ and IL-10 differentiated patients in the early stages of GC. Still, we caution that studies with larger groups of patients and properly matched control groups, are needed to confirm these findings. When evaluating candidate biomarkers in cancer, it is important to analyze their utility as a diagnostic test, as showed in this study.

\section{Additional files}

Additional file 1: Table S1. Number of cases included for the different cytokines and chemokines. Table S2. Estimated cut off values and area under the curve for each cytokine and chemokine using a ROC analyses. (DOC 33 kb)

Additional file 2: Fig. S1. The concentrations of IL-8 and TNF-a in healthy controls and gastric cancer patients do not differ. Circulating concentrations of IL-8 (A and $\mathbf{B}$ ) and TNF- $\mathbf{A}(\mathbf{C})$ in healthy donors and patients with gastric cancer were measured by XMAP. All measurements were made in duplicate. The statistical analysis was performed by Mann-Whitney $U$ test and results for each group are presented as median with interquartile range. (TIFF $63 \mathrm{~kb}$ )

Additional file 3: Tabular data provided in format CSV. Data File. All data generated or analysed during this study are included in this file. (CSV 65 kb)

\section{Abbreviations \\ Cl: Confidence interval; COX-2: Cyclooxygenase-2; DGC: Diffuse gastric cancer; EDTA: Ethylenediaminetetraacetic acid; ELISA: Enzyme-linked immunosorbent assay; GC: Gastric cancer; HIF-1: Hypoxia-inducible factor 1; IFN- $\gamma$ : Interferon gamma; IGC: Intestinal gastric cancer; IL: Interleukin; MCP- 1: Monocyte chemoattractant protein-1; MMP: Matrix metalloproteinase; NPV: Negative predictive value; OR: Odds ratio; PPV: Positive predictive value; ROC: Receiver-operating characteristic; STAT3: Signal transducer and activator of transcription 3; TGF: Transforming growth factor beta; TNF-a: Tumor necrosis factor alpha; TNM: Tumor-node-metastasis; VEGF: Vascular endothelial growth factor; XMAP: Multiplex analyte profiling technology}

\section{Acknowledgments}

SZN thanks the Program of "Doctorado en Ciencias Químico-Biológicas" of the Escuela Nacional de Ciencias Biológicas del Instituto Politécnico Nacional. This work constitutes a partial fulfillment of the requirements for the Graduate Program. She also thanks CONACYT Mexico for providing a fellowship during her doctoral studies. 


\section{Funding}

This work was supported by CONACYT-FOSISS, MEXICO [SALUD-2007-0169,450], by Instituto de Ciencia y Tecnología del Distrito Federal [ICYTDF/ 234/2012 (PICSA 12-209)], and by the Hospital Infantil de México Federico Gómez (HIM/2012/018). There was not any role of the funding body in the design of the study and collection, analysis, and interpretation of data and in writing the manuscript.

\section{Availability of data and materials}

Raw data are provided in Additional file 3.

\section{Authors' contributions}

MBC and JT designed and coordinated the study and helped draft the manuscript. SZN participated in the design of the study, performed all cytokine analyses, built the database study, and drafted the manuscript. AG performed all statistical analyses. CPM and PML performed all bacteriological and serological tests to identify H. pylori. HGR and MGR identified and selected all patients with gastric cancer for inclusion in the study. GCS, SZN, AG, MBC, and JT analyzed all results of the study. All authors read and approved the final manuscript.

\section{Competing interests}

The authors declare that they have no competing interests.

\section{Consent for publication}

Not applicable.

\section{Ethics approval and consent to participate}

Informed written consent was obtained from all patients prior to enrollment in the study. The Ethics Committees of the two participating institutions, IMSS and SS, approved the study. Instituto Mexicano del Seguro Social. Registry Number (Minister of Health, Mexico) $13 \mathrm{Cl} 09015213$ and Secretaría de Salud, COFEPRIS 083300538B0047, OHRP FWA00013098.

\section{Publisher's Note}

Springer Nature remains neutral with regard to jurisdictional claims in published maps and institutional affiliations.

\section{Author details}

${ }^{1}$ Laboratorio de Investigación en Inmunología y Proteómica, Hospital Infantil de México Federico Gómez, Dr. Márquez 162, Col. Doctores, 06720 Mexico City, Mexico. ${ }^{2}$ División de Auxiliares de Diagnóstico y Tratamiento UMAE Hospital de Especialidades, Centro Médico Nacional-Siglo XXI, IMSSI, Avenida Cuauhtémoc 330, Col Doctores, 06720 Mexico City, Mexico. ${ }^{3}$ Laboratorio de Bacteriología, Escuela Nacional de Ciencias Biológicas-IPN, Prolongación Manuel Carpio y Plan de Ayala, Santo Tomás, 11350 Mexico City, Mexico. ${ }^{4}$ Unidad de Investigación en Enfermedades Infecciosas, Hospital de Pediatría, Centro Médico Nacional Siglo XXI, IMSS, Avenida Cuauhtémoc 330, Col Doctores, 06720 Mexico City, Mexico. ${ }^{5}$ Departamento de Patología, Instituto Nacional de Cancerología, Secretaría de Salud, Av. San Fernando 22, Tlalpan, 1408 Mexico City, Mexico. ${ }^{6}$ Departamento de Sarcomas, Tracto Digestivo Bajo, UMAE Oncología, Centro Médico Nacional Siglo XXI, IMSS, Av. Cuauhtémoc 330, Col Doctores, 06720 Mexico City, Mexico.

Received: 12 September 2016 Accepted: 1 May 2017

Published online: 30 May 2017

\section{References}

1. International Agency for Research on Cancer. GLOBOCAN 2012: estimated cancer incidence, mortality, and prevalence worldwide in 2012. 2012. http://globocan.iarc.fr. Accessed 18 May 2017.

2. Talaiezadeh A, Hajiani E, Tarshizi MA. The relative frequency of the Helicobacter pylori infection in proximal gastric cancers. Pol Przegl Chir. 2013;85(11):657-62.

3. Kamangar F, Dawsey SM, Blaser MJ, Perez-Perez GI, Pietinen P, Newschaffer CJ, et al. Opposing risks of gastric cardia and noncardia gastric adenocarcinomas associated with Helicobacter pylori seropositivity. J Natl Cancer Inst. 2006;98: 1445-52.

4. Hamajima N, Goto Y, Nishio K, Tanaka D, Kawai S, Sakakibara H, et al. Helicobacter pylori eradication as a preventive tool against gastric cancer. Asian Pac J Cancer Prev. 2004;5(3):246-52.
5. Peek RM Jr, Fiske C, Wilson KT. Role of innate immunity in Helicobacter pylori-induced gastric malignancy. Physiol Rev. 2010;90:831-58.

6. Israel DA, Peek RM. Pathogenesis of Helicobacter pylori-induced gastric inflammation. Aliment Pharmacol Ther. 2001;5:1271-90.

7. Borrello MG, Degl'Innocenti D, Pierotti MA. Inflammation and cancer: the oncogene-driven connection. Cancer Lett. 2008;267:262-70.

8. Lu J, Huang CM, Zheng CH, Li P, Xie JW, Wang JB, et al. Prognostic value of tumor size in patients with remnant gastric cancer: is the seventh UICC stage sufficient for predicting prognosis? PLoS One. 2014;9:e115776

9. Vauhkonen M, Vauhkonen $\mathrm{H}$, Sipponen P. Pathology and molecular biology of gastric cancer. Best Pract Res Clin Gastroenterol. 2006;20:651-74.

10. Sistema Nacional de Información en Salud, Sistema Nacional de Vigilancia Epidemiológica, Dirección General de Epidemiología, Secretaría de Salud. Perfil epidemiológico de los tumores malignos en México. Ciudad de México: Secretaria de Salud, Mexico; 2011. http://docplayer.es/11449544-Perfilepidemiologico-de-los-tumores-malignos-en-mexico.html. Accessed 18 May 2017.

11. IARC Helicobacter pylori Working Group (2014). Helicobacter pylori Eradication as a Strategy for Preventing Gastric Cancer. Lyon, France: International Agency for Research on Cancer (IARC Working Group Reports, No. 8). Available from: http://www.iarc.fr/en/publications/pdfs- online/wrk/wrk8/ index.php. Accessed 18 May 2017.

12. Martínez-Galindo MG, Zamarripa-Dorsey F, Carmona-Castañeda A, Angeles-Labra A, Peñavera-Hernández R, Ugarte-Briones C, et al. Histopathologic characteristics of gastric adenocarcinoma in Mexican patients: a 10-year experience at the Hospital Juárez of Mexico. Rev Gastroenterol Mex. 2015;80:21-6.

13. Hitzler I, Sayi A, Kholer E, Engler DB, Koch KN, Hardt WD, et al. Caspase-1 has both proinflammatory and regulatory properties in Helicobacter infections, which are differentially mediated by its substrates IL-1 $\beta$ and IL-18. J Immunol. 2012;188:3594-602.

14. Tu S, Bhagat G, Cui G, Takaishi S, Kurt-Jones EA, Rickman B, et al. Overexpression of interleukin-1 $\beta$ induces gastric inflammation and cancer and mobilizes myeloid-derived suppressor cells in mice. Cancer Cell. 2008;14:408-19.

15. McLean MH, El-Omar EM. Genetics of gastric cancer. Nat Rev Gastroenterol Hepatol. 2014;11:664-74.

16. Ashizawa T, Okada R, Suzuki Y, Takagi M, Yamazaki T, Sumi T, et al. Clinical significance of interleukin-6 (IL-6) in the spread of gastric cancer: role of IL-6 as a prognostic factor. Gastric Cancer. 2005;8:124-31.

17. Szczepanik AM, Scislo L, Scully T, Walewska E, Siedlar M, Kolodziejczyk P, et al. IL-6 serum levels predict postoperative morbidity in gastric cancer patients. Gastric Cancer. 2011;14:266-73.

18. Foran E, Garrity-Park MM, Mureau C, Newell J, Smyrk TC, Limburg PJ, et al. Upregulation of DNA methyltransferase-mediated gene silencing, anchorage-independent growth, and migration of colon cancer cells by interleukin-6. Mol Cancer Res. 2010;8:471-81.

19. Grivennikov S, Karin E, Terzic J, Mucida D, Yu GY, Vallabhapurapu S, et al. IL-6 and Stat3 are required for survival of intestinal epithelial cells and development of colitis-associated cancer. Cancer Cell. 2009;15:103-13

20. Wang Z, Si X, Xu A, Meng X, Gao S, Qi Y, et al. Activation of STAT3 in human gastric cancer cells via interleukin (IL)-6-type cytokine signaling correlates with clinical implications. PLoS One. 2013;8:e75788.

21. Lindgren $\AA$, Yun CH, Sjöling A, Berggren C, Sun JB, Jonsson E, et al. Impaired IFN- $\gamma$ production after stimulation with bacterial components by natural killer cells from gastric cancer patients. Exp Cell Res. 2011;317:849-58.

22. Shankaran V, Ikeda H, Bruce AT, White JM, Swanson PE, Old L, et al. IFNY and lymphocytes prevent primary tumour development and shape tumour immunogenicity. Nature. 2001;410:1107-11.

23. Mohammadi M, Czinn S, Redline R, Nedrud J. Helicobacter-specific cellmediated immune responses display a predominant Th1 phenotype and promote a delayed-type hypersensitivity response in the stomach of mice. J Immunol. 1996;156(12):4729-38.

24. Mohammadi M, Nedrud J, Redline R, Lycke N, Czinn SJ. Murine CD4 T-cell response to Helicobacter infection: Th1 cells enhance gastritis and Th2 cells reduce bacterial load. Gastroenterology. 1997;113:1848-57.

25. Tu SP, Quante M, Bhagat G, Takaishi S, Cui G, Yang XD, et al. IFN- $\gamma$ inhibits gastric carcinogenesis by inducing epithelial cell autophagy and T-cell apoptosis. Cancer Res. 2011;71:4247-59.

26. Xue H, Lin B, An J, Zhu Y, Huang G. Interleukin-10-819 promoter polymorphism in association with gastric cancer risk. BMC Cancer. 2012;12:102. 
27. Fortis C, Foppoli M, Gianotti L, Galli L, Citterio G, Consogno G, et al. Increased interleukin 10 serum levels in patients with solid tumours. Cancer Lett. 1996;104:1-5.

28. Szkaradkiewicz A, Karpiński TM, Drews M, Borejsza-Wysocki M, Majewski P, Andrzejewska E. Natural killer cell cytotoxicity and immunosuppressive cytokines (IL-10, TGF- $\beta 1)$ in patients with gastric cancer. J Biomed Biotechnol. 2010;2010:901564.

29. Tonouchi H, Miki C, Tanaka K, Kusunoki M. Profile of monocyte chemoattractant protein-1 circulating levels in gastric cancer patients. Scand J Gastroenterol. 2002;37:830-3.

30. Shimoyama T, Everett SM, Dixon MF, Axon AT, Crabtree JE. Chemokine mRNA expression in gastric mucosa is associated with Helicobacter pylori cagA positivity and severity of gastritis. J Clin Pathol. 1998;51:765-70.

31. Salcedo R, Ponce ML, Young HA, Wasserman K, Ward JM, Kleinman HK, et al. Human endothelial cells express CCR2 and respond to MCP-1: direct role of MCP-1 in angiogenesis and tumor progression. Blood. 2000;96(1):34-40.

32. Saji H, Koike M, Yamori T, Saji S, Seiki M, Matsushima K, et al. Significant correlation of monocyte chemoattractant protein-1 expression with neovascularization and progression of breast carcinoma. Cancer. 2001;92:1085-91.

33. Epplein M, Xiang YB, Cai Q, Peek RM Jr, Li H, Correa P, et al. Circulating cytokines and gastric cancer risk. Cancer Causes Control. 2013;24:2245-50.

34. Kuai WX, Wang Q, Yang XZ, Zhao Y, Yu R, Tang XJ. Interleukin-8 associates with adhesion, migration, invasion and chemosensitivity of human gastric cancer cells. World J Gastroenterol. 2012;18:979-85.

35. Matsuo Y, Ochi N, Sawai H, Yasuda A, Takahashi H, Funahashi H, et al. CXCL8/IL-8 and CXCL12/SDF-1 a co-operatively promote invasiveness and angiogenesis in pancreatic cancer. Int J Cancer. 2009;124:853-61.

36. Lee KE, Khoi PN, Xia Y, Park JS, Joo YE, Kim KK, et al. Helicobacter pylori and interleukin-8 in gastric cancer. World J Gastroenterol. 2013;19:8192-202.

37. Sethi $G$, Sung B, Aggarwal BB. TNF-a master switch for inflammation to cancer. Front Biosci. 2008;13:5094-107.

38. Wu Y, Li Q, Zhou X, Yu J, Mu Y, Munker S, et al. Decreased levels of active SMAD2 correlate with poor prognosis in gastric cancer. PLoS One. 2012; : e35684.

39. Diamandis EP. Cancer biomarkers: can we turn recent failures into success? J Natl Cancer Inst. 2010;102:1462-7.

\section{Submit your next manuscript to BioMed Central and we will help you at every step:}

- We accept pre-submission inquiries

- Our selector tool helps you to find the most relevant journal

- We provide round the clock customer support

- Convenient online submission

- Thorough peer review

- Inclusion in PubMed and all major indexing services

- Maximum visibility for your research

Submit your manuscript at www.biomedcentral.com/submit

) Biomed Central 